\title{
Circulating LncRNAs Serve as Diagnostic Markers for Hepatocellular Carcinoma
}

\author{
Weidong Yuan Yong Sun Ling Liu Bing Zhou Shuming Wang Dianhua Gu \\ Department of Hepatobiliary Surgery, Huai'an First People's Hospital, Huai'an, Jiangsu, P. R. China
}

\author{
Key Words \\ HcC $・$ Fingerprint $\cdot \mathrm{AUC} \cdot \operatorname{LnCRNA} \cdot$ Plasma
}

\begin{abstract}
Background/Aims: Circulating (serous or plasmic) long non-coding RNA (IncRNA) as biomarkers for predicting the diagnosis or prognosis of human disease have been well documented. Due to the sensibility or specificity limitation of Alpha Fetoprotein (AFP), a cluster IncRNAs were revealed as fingerprints for hepatocellular carcinoma (HCC). In this study, we enrolled all the reported circulating IncRNAs in HCC as candidate targets and examined in an independent cohort. Methods: The candidate IncRNAs were determined by qRT-PCR divided into training and validation sets. The risk score analysis was employed to evaluate the potential diagnosis ability of the IncRNAs independently or combining with AFP value. The receiver operating characteristic curve (ROC) was applied for presentation of sensibility or specificity. Results: Among the ten candidate circulating IncRNA, LINC00152, RP11-160H22.5, XLOC014172 and LOC149086 were screened with significant difference in training set. Further investigation in validation set indicated LINC00152, RP11-160H22.5 and XLOC014172 might be the fingerprints for $\mathrm{HCC}$ comparing with chronic hepatitis $(\mathrm{CH})$ patients or healthy controls. The risk score analysis revealed the combination of three IncRNAs with AFP could distinguish the $\mathrm{HCC}$ from either $\mathrm{CH}$ or healthy control with the area under curve value (AUC) of 0.986 and 0.985 , respectively. Conclusion: The three IncRNAs may act as novel biomarkers for acting as fingerprint in HCC combining with AFP.

\section{Introduction}

As one of the most common life-threatening malignancies worldwide, hepatocellular carcinoma (HCC) was estimated to cause 748, 300 new liver cancer cases and 695, 900 cancer deaths worldwide per year and nearly half of these cases and deaths were estimated to take place in China [1-3]. Although the major risk factors have been identified, including hepatitis C (HCV) and B virus (HBV) infection, toxins (aflatoxin B1), chronic alcohol abuse and nonalcoholic fatty liver disease [4-6]. The early diagnosis of HCC still remain a crucial issue currently due the low sensibility or specificity limitation of Alpha Fetoprotein (AFP)[7-9]. Hepatitis B virus (HBV) infection is an important factor for HCC occurrence. The association 
between HBV and HCC has been demonstrated by multiple epidemiological studies: High HBV prevalence may result in a high frequency of occurrence of HCC, and the risk of HCC in HBV carriers is significantly increased compared with that of non-carriers [10]. Thus, the occurrence of hepatocellular carcinoma (HCC) is decreased but not eliminated. The molecular mechanisms as well as the biomarkers for HCC that contributing to the occurrence and development of HCC remain elusive, hindering the development of clinic diagnostic or therapeutic approaches.

To date, the long non-coding has been proved as important role in the pathogenesis of human disease including HCC[11-13]. LncRNAs were considered as non-protein-coding transcripts longer than 200 nucleotides and widely expressed in various organs and tissues of the body [14-16]. Functional lncRNA was involved in various cancer biological processes, such as proliferation, apoptosis, invasion and metastasis through modulation of chromatin remodeling, epigenetics modification, regulation of gene transcription, post-transcriptional mRNA processing, protein function and localization, and intercellular signal transduction regulation [17]. Many previous studies showed that lncRNAs might be as biomarkers in multiple cancers. For example, The MD-miniRNA, derived from MALAT-1, has been explored, and was available for clinical detection for human prostate cancer [18]. The fragment of Linc00974 could was also detectable and might improve the prognosis of HCC by comparing with CYFRA-21-1[19].

In this study, we conducted our experiment based on the identified lncRNAs which was certain diagnostic value in HCC according to previous report. We enrolled ten candidate lncRNAs for screening. We hypothesized that these HCC-related lncRNAs might be utilized to detect and early monitor HCC by combining with the well-known maker AFP. We aimed to explore the potential lncRNA acting as fingerprint for the early identification of HCC.

\section{Materials and Methods}

Samples and Screening phase

100 fresh HCC plasma samples were obtained from the patients received therapy at Huai'an First People's Hospital. Controls were from healthy volunteers without any health problems during their health check-ups at the Huai'an First People's Hospital. The study was approved by our Institutional Ethics Committee of Huai'an First People's Hospital.

All patients provided their written informed consent to participate in the study. The HCC patients were confirmed by the histopathological examination. Peripheral blood samples of patients were collected before surgery. Blood samples were collected in a separate vacuum cube, followed by centrifugation at 3, $000 \mathrm{rpm}$ for $10 \mathrm{~min}$. All samples were stored at $-80^{\circ} \mathrm{C}$ until further analysis. The clinical characteristics of all patients are summarized in Table 1. All candidates were tested in an independent cohort of 20 plasma samples obtained from patients regarding as training set. The rest 80 samples was labelled as validation set randomly.

Table 1. Clinicopathological features analysis of hepatocellular carcinoma (HCC) patients, patients with HBV-positive chronic hepatitis (CH) and cancer-free control samples. ${ }^{a}$ Student t-test. ${ }^{b}$ Chi-square test

\begin{tabular}{lcccc}
\hline & HCC & CH & Control & P value \\
\hline $\mathrm{N}$ & 100 & 100 & 100 & \\
Age Mean (SE) year & $54.31(0.36)$ & $53.98(0.51)$ & $54.13(0.42)$ & $0.37^{\mathrm{a}}$ \\
Sex (male/female) & $87 / 13$ & $85 / 15$ & $88 / 12$ & $0.22^{\mathrm{b}}$ \\
Smoking history & & & & \\
No & 23 & 31 & 67 & $<0.001^{\mathrm{b}}$ \\
Yes & 77 & 69 & 33 & \\
Tumor Size(cm) & & & & \\
$\leq 3 \mathrm{~cm}$ & 66 & & & \\
$>3 \mathrm{~cm}$ & 34 & & & \\
Tumor Number & & & & \\
Solitary & 41 & & & \\
Multiple & 59 & & & \\
Tumor Capsular & & & & \\
Incomplete & 32 & & & \\
Complete & 68 & & & \\
TNM stage & & & & \\
I-II & 71 & & & \\
III-IV & 29 & & & \\
AFP(ng/ml) & & & & \\
$<20$ & 11 & & & \\
$\geq 20$ & 89 & 65 & & \\
\hline
\end{tabular}




\section{Cellular Physiology Cell Physiol Biochem 2017;44:125-132 and Biochemistry Published online: November 07, 2017 \begin{tabular}{l|l} 
DOI: 10.1159/000484589 2017 The Author(s). Published by S. Karger AG, Basel \\
www.karger.com/cpb
\end{tabular} \\ Yuan et al.: Plasma LncRNA in HCC}

\section{Quantitative real-time PCR (qRT-PCR)}

Total RNAs were obtained from plasmic samples using TRIzol reagent as described by the manufacturer (Invitrogen Life Technologies Co, Carlsbad, CA, USA). For mRNA detection, total RNAs (500ng) were reverse transcribed using the reverse transcription kit (Takara, Tokyo, Japan). The quality of RNA samples was assessed by a UV spectrophotometer (Bio-Rad, Hercules, CA, USA), and the 260/280nm absorbance ratio of samples were limited to 1.8-2.0. QRT-PCR was performed by using ABI Prism 7900HT (Applied Biosystems, CA, USA). The expression levels of these candidates were analyzed in all samples, and comparative $2^{-\Delta \Delta C t}$ method algorithms were used to analyze the difference between patients and health controls.

\section{Risk score analysis}

Risk score analysis was performed to evaluate the associations between the concentrations of the plasma IncRNA expression levels. The upper 95\% reference interval of each IncRNA value in controls or $\mathrm{CH}$ group was set as the threshold to code the expression level of the corresponding lncRNA for each sample as 0 and 1 in the training set. A risk score function (RSF) to predict HCC group was defined according to a linear combination of the expression level for each IncRNA. For example, the RSF for sample i using information from three lncRNAs was: $r s f i=\sum 3 j-1 W j . s i j$. In the above equation, sij is the risk score for IncRNA $j$ on sample $i$, and $W_{j}$ is the weight of the risk score of IncRNA $j$. The risk score of three lncRNAs was calculated using the weight by the regression coefficient that was derived from the univariate logistic regression analysis of each IncRNAs. Samples were ranked according to their RSF and then divided into a high-risk group, representing the HCC patients, and a low-risk group, representing the predicted control individuals or $\mathrm{CH}$ patients. Frequency tables and ROC curves were then used to evaluate the diagnostic effects of the profiling and to find the appropriate cutoff point, and to validate the procedure and cutoffs in the next validation sample set.

\section{Statistical analysis}

Data were presented as mean (S.E.M.). The Student's t-test and Mann-Whitney unpaired test analysis of variance were used to evaluate statistical differences between patients and controls. Analysis of area under the ROC curve (AUC) was used to estimate the effectiveness of IncRNA. Statistical analysis was performed using softwares of STATA 10.0 and GraphPad prism. In all cases, $P<0.05$ was considered to be significant. All $P$ values were two-sided.

\section{Results}

Screening the HCC-associated IncRNAs in training set

The ten candidate circulating lncRNAs was enrolled including DANCR, SPRY4-IT1, HULC, LINC00152, LINC00974, MALAT1, LncRNA-CTBP, RP11-160H22.5, XLOC014172 and LOC149086. The candidates were first detected in the 20 plasma samples as training set. We found that the four lncRNAs (LINC00152, RP11-160H22.5, XLOC014172 and LOC149086) were significant different in HCC patients comparing with either patients with $\mathrm{CH}$ or healthy control (Fig. 1)

\section{Confirming the candidate biomarker in validation set}

The following investigation was conducted by detecting the expression of the four lncRNAs in the validation set. As presented in Fig. 2, LINC00152, RP11-160H22.5 and XLOC014172 were confirmed in the larger individual samples indicating as the potential biomarker for predicting HCC, while the LOC149086 present no significant difference.

\section{Diagnostic potency prediction by risk score analysis}

Researcher has reported the three IncRNAs might be potential biomarkers for HCC; however, they only focused on predictive value of these lncRNAs through healthy controls, no evidence was given for these IncRNAs to predict the HCC from patients with $\mathrm{CH}$. This might be a useful factor for clinical application. Here, in this study, to validate the accuracy and specificity of these 3 lncRNA as a HCC potential signature, the risk score formula was applied to assess the diagnostic value of the three lncRNAs profiling system by combining with AFP. 
Fig. 1. Relative expression of ten candidate IncRNAs in training set. The 20 paired plasma from HCC patients and 20 cancer-free controls were applied by using RTqPCR. Data was presented as mean \pm SEM. Data was analyzed by student $t$ test. $*$ indicated $\mathrm{p}<0.05, * *$ indicated $\mathrm{p}<0.01$.

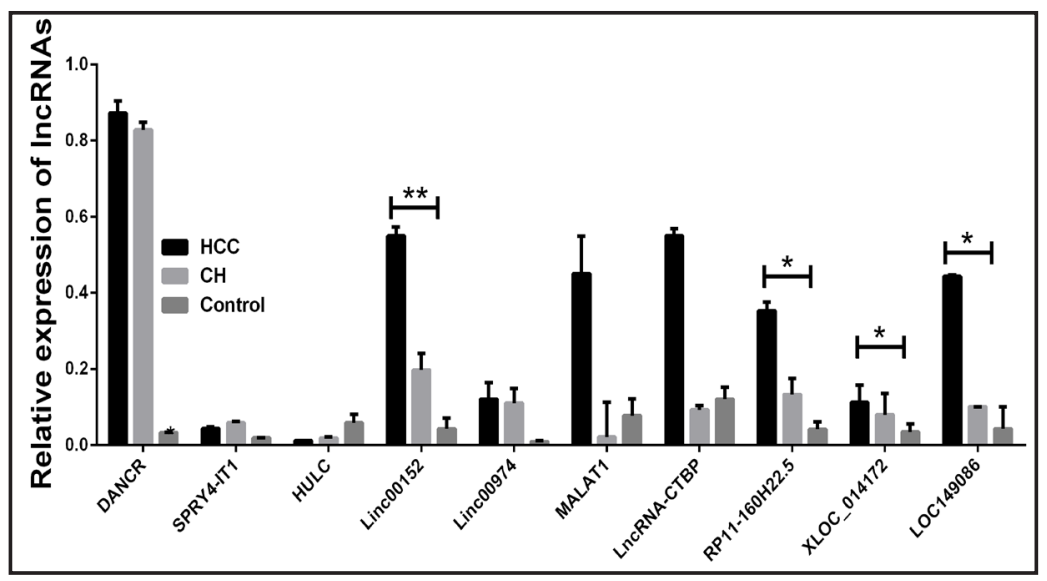

Fig. 2. Relative expression of lncRNAs in validation set. Data were presented as plot of the median and range of log-transformed relative expression level and was analyzed with student $\mathrm{t}$ test. ${ }^{* *}$ indicated $\mathrm{p}<0.01$.

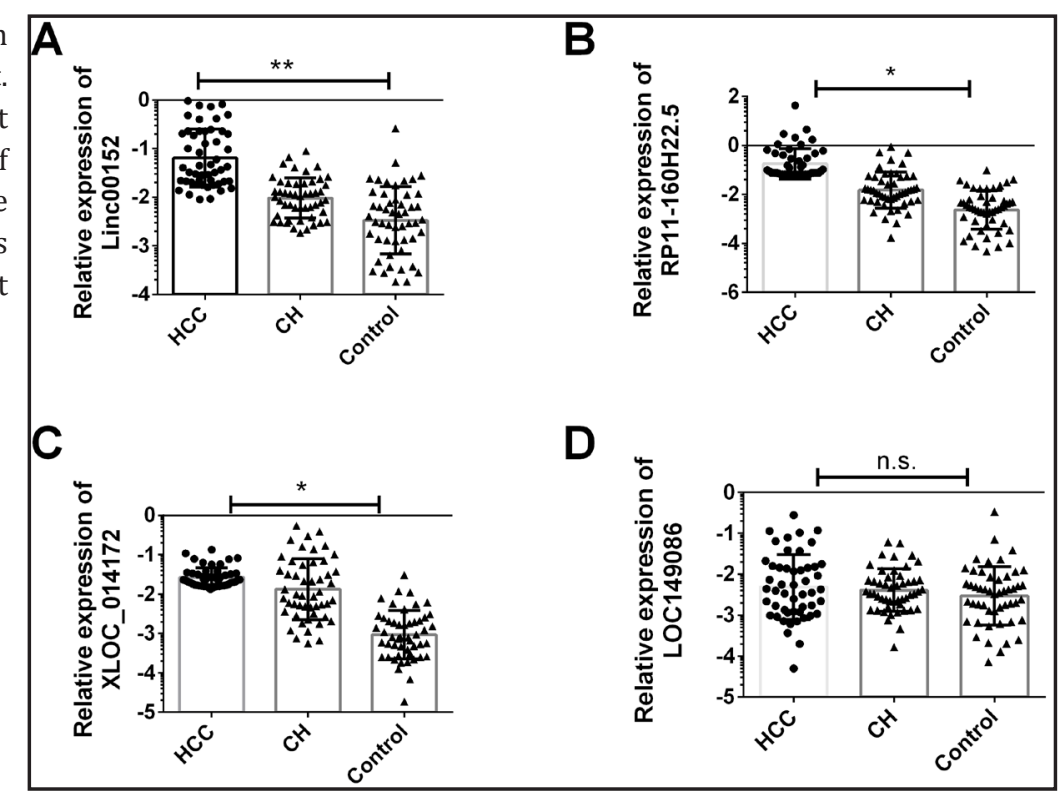

Table 2. Risk score analysis of in HCC and cancer-free

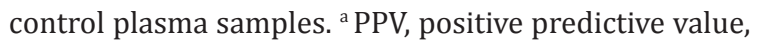
${ }^{\mathrm{b}} \mathrm{NPV}$, negative predictive value

\begin{tabular}{lcccc} 
Score & $0-8.12$ & $8.12-13.31$ & PPV a & NPVb \\
\hline Training set & & & 0.70 & 0.80 \\
HCC & 6 & 14 & & \\
Control & 16 & 4 & & \\
Validation set & & & 0.88 & 0.81 \\
HCC & 12 & 88 & & \\
Control & 81 & 19 & & \\
\hline
\end{tabular}

First, we analyzed the predictive value of these three lncRNA and AFP comparing with the healthy control. The risk score of each plasma sample in the training set was calculated, as the basis of their risk scores and a set cut off, plasma samples were then divided into a high-risk group, representing the possible HCC group, and a low-risk group, representing the predicted controls. At the optimal cutoff value (Value $=8.12$ ) with the value of sensitivity + specificity considered to be maximal, the positive predictive value (PPV) and negative predictive value (NPV) was $70 \%$ and $80 \%$ in the training set, respectively. Similarly, when the same cutoff value was applied to calculate the risk score of samples in the larger validation sets, the PPV and NPV were $88 \%, 81 \%$, respectively (Table 2). ROC curve analysis was used to evaluate the predicting diagnosis value of lncRNAs and AFP for HCC. Areas under the ROC curves of the validated three IncRNAs signature were $0.869,0.884$ and 0.759 , respectively while the combination of the three factors with AFP possessed a moderate ability for discrimination between HCC patients and controls with an area under the ROC curve of 0.986 (Fig. 3A). 


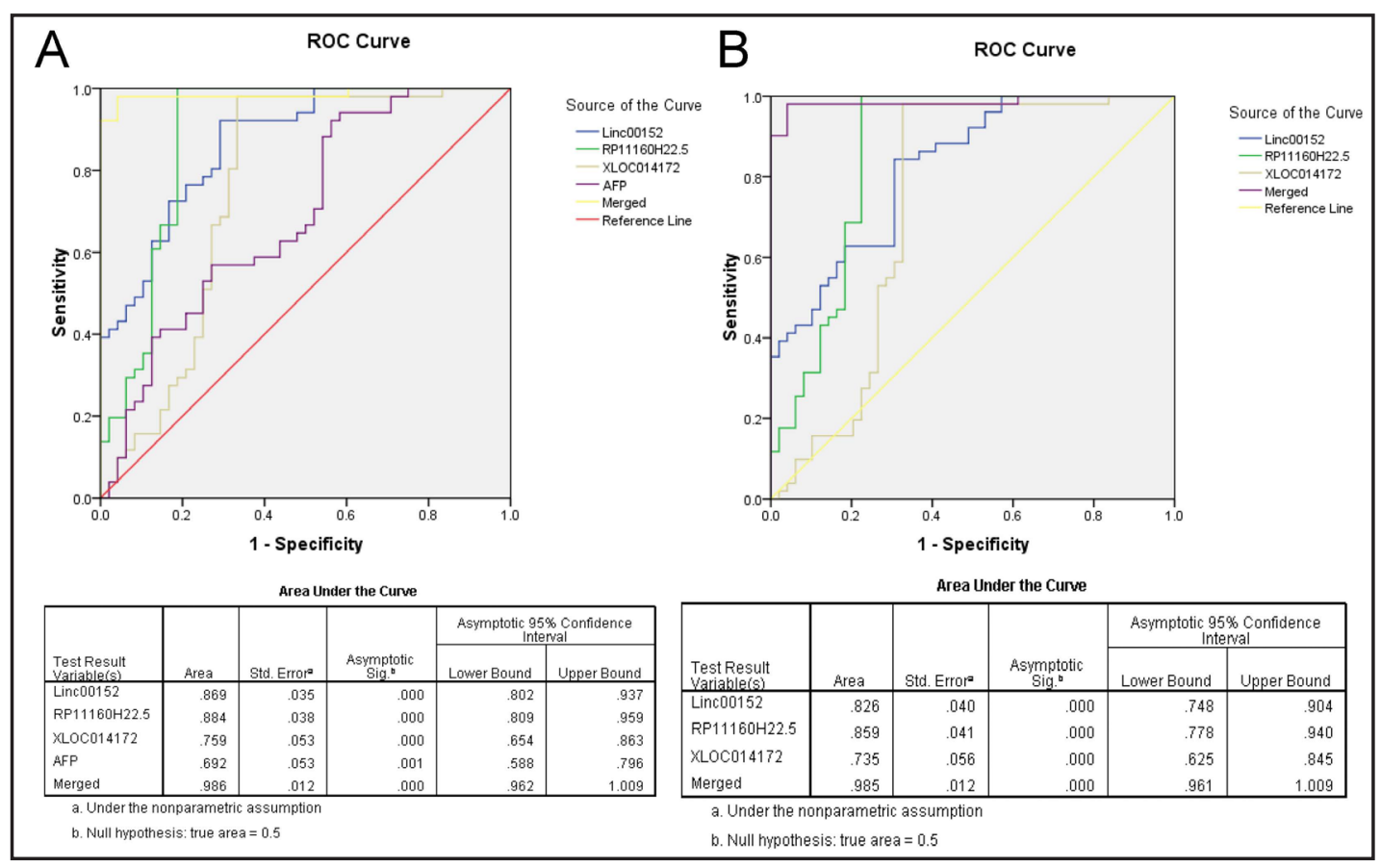

Fig. 3. ROC analysis of the three potential biomarkers for HCC by using risk score analysis combining with AFP. A: The ROC analysis of lncRNAs combining with AFP to predict HCC from healthy controls. B: The ROC analysis of lncRNAs to predict HCC from patient with $\mathrm{CH}$.

Table 3. Risk score analysis of in HCC and CH control plasma samples. ${ }^{a} \mathrm{PPV}$, positive predictive value, ${ }^{\mathrm{b}}$ NPV, negative predictive value

\begin{tabular}{lcccc}
\hline Score & $0-5.88$ & $5.88-11.25$ & PPV a & NPVb \\
Training set & & & 0.65 & 0.80 \\
HCC & 7 & 13 & & \\
CH & 16 & 4 & & \\
Validation set & & & 0.87 & 0.80 \\
HCC & 13 & 87 & & \\
CH & 80 & 20 & & \\
\hline
\end{tabular}

The similar strategy was applied for predicting the HCC diagnostic value of lncRNAs by comparing with patients with $\mathrm{CH}$. At the optimal cutoff value (Value=5.88) with the value of sensitivity + specificity considered to be maximal, the positive predictive value (PPV) and negative predictive value (NPV) was $65 \%$ and $80 \%$ in the training set, respectively. Similarly, when the same cutoff value was applied to calculate the risk score of samples in the larger validation sets, the PPV and NPV were 87\%, 80\%, respectively (Table 3). ROC curve analysis was used to evaluate the predicting diagnosis value of lncRNAs and AFP for HCC. Areas under the ROC curves of the validated three lncRNAs signature were $0.826,0.859$ and 0.735 , respectively While the combination of the three factors possessed a moderate ability for discrimination between HCC patients and controls with an area under the ROC curve of 0.985 (Fig. 3B).

\section{Discussion}

Hepatocellular carcinoma (HCC) is the sixth most common malignant tumor with the third leading cause of cancer-related death in the world [20, 21]. HCC is chartered by high malignancy, easy invasion especially towards intrahepatic invasion and frequent reoccurrence after surgical resection [22]. Increasing evidence indicated IncRNAs as biomarker for predicting survival and metastasis or diagnosing in multiple diseases which was confirmed contributing to many biological processes through diverse mechanisms [11]. 


\section{Cellular Physiology Cell Physiol Biochem 2017;44:125-132 \begin{tabular}{l|l|l} 
and Biochemistry 10.1159/000484589 & $\begin{array}{l}\text { C) } 2017 \text { The Author(s). Published by S. Karger AG, Basel } \\
\text { www.karger.com/cpb }\end{array}$ \\
\hline Published online: November 07, 2017
\end{tabular} \\ Yuan et al.: Plasma LncRNA in HCC}

Mechanism study have been described for selected lncRNA involved in liver diseases include widely diverse functions such as DNA imprinting, $\mathrm{X}$ inactivation, DNA demethylation, gene transcription and generation of other RNA molecules [23].

Not only the miRNA, but also the lncRNA have been annotated acting as biomarkers in predicting the feature of tumor. For example, circulating serum lncRNA RP11-445H22.4 as biomarker for the detection of breast cancer [24]. In this study, the ten IncRNA was enrolled as candidates based on the important function reported previously. These tissues different expressed lncRNA might present an alternated level in patients' bodily fluid. Research has speculated that the aberrant expressed circulation lncRNAs might be derived from the tumor. Thus, we examined the expression of the 10 lncRNAs in patients and healthy control. Accordingly, Long noncoding RNA DANCR could increase stemness features of hepatocellular carcinoma by derepression of CTNNB1[25]. Overexpression of the long noncoding RNA SPRY4-IT1 promotes tumor cell proliferation and invasion by activating EZH2 in hepatocellular carcinoma [26]. HULC and Linc00152 Act as Novel Biomarkers in Predicting Diagnosis of Hepatocellular Carcinoma [27]. A novel biomarker Linc00974 interacting with KRT19 promotes proliferation and metastasis in hepatocellular carcinoma [19]. Long NonCoding RNA MALAT1 Regulates ZEB1 Expression by Sponging miR-143-3p and Promotes Hepatocellular Carcinoma Progression [28]. The lncRNA-CTBP has also been identified had high sensitivity and specificity for discriminating HCC from healthy controls and also from $\mathrm{CH}$ patients [29]. Tang et al. also published the results that lncRNAs might act as biomarker for the diagnosis of HCC from healthy control and $\mathrm{CH}$ patients [30]. In this study, by employing a multiple screening phase, we found that LINC00152, RP11-160H22.5, XLOC014172 and LOC149086 were screened with significant difference in training set. Further investigation in validation set indicated LINC00152, RP11-160H22.5 and XLOC014172 might be the fingerprints for HCC comparing with patients with chronic hepatitis $(\mathrm{CH})$ or healthy controls.

For the biomarkers screening study in HCC, researchers has identified multiple factors that might be the potential fingerprint; however, most of this study was conducted by comparing the expression of these IncRNAs in a case-control study. The control group enrolled was healthy volunteers. This kind of biomarker might be useful for distinguish the HCC patients from healthy human; however, the HCC patients was developed from HBV positive patients, most of them might be suffering with $\mathrm{CH}$ or liver cirrhosis. Biomarkers for predicting $\mathrm{HCC}$ from patients with $\mathrm{CH}$ or liver cirrhosis might be useful for clinical approach especially for the early diagnosis of HCC.

Researchers has found that cigarette smoke exposure will cause an aberrant expression profiling of lncRNA in lung cancer associated cell lines [31]. Based on the different cigarette exposure in the three groups, we thought it was necessary to identify the expression of circulating lncRNA in patients with the cigarette exposure or not. This might give us a novel point both for the pathogenesis and diagnosis of HCC.

\section{Conclusion}

We identified three lncRNAs, LINC00152, RP11-160H22.5 and XLOC014172, as the potential biomarker for the tumorigenesis prediction of HCC from both healthy controls and patients with $\mathrm{CH}$ combining with AFP in this study. These factors might serve as a better biomarker for the early diagnosis of HCC especially for patients with HBV infection background.

\section{Disclosure Statement}

The authors declare that they have no financial conflict of interest. 


\section{Cellular Physiology Cell Physiol Biochem 2017;44:125-132 and Biochemistry Published online: November 07, 2017 \begin{tabular}{l|l} 
DOI: 10.1159/000484589 2017 The Author(s). Published by S. Karger AG, Basel \\
www.karger.com/cpb
\end{tabular} \\ Yuan et al.: Plasma LncRNA in HCC}

\section{References}

1 Chaisaingmongkol J, Budhu A, Dang H, Rabibhadana S, Pupacdi B, Kwon SM, Forgues M, Pomyen Y, Bhudhisawasdi V, Lertprasertsuke N, Chotirosniramit A, Pairojkul C, Auewarakul CU, Sricharunrat T, Phornphutkul K, Sangrajrang S, Cam M, He P, Hewitt SM, Ylaya K, Wu X, Andersen JB, Thorgeirsson SS, Waterfall JJ, Zhu YJ, Walling J, Stevenson HS, Edelman D, Meltzer PS, Loffredo CA, Hama N, Shibata T, Wiltrout RH, Harris CC, Mahidol C, Ruchirawat M, Wang XW, Consortium T-L: Common Molecular Subtypes Among Asian Hepatocellular Carcinoma and Cholangiocarcinoma. Cancer Cell 2017;32:57-60.

- 2 Logan GJ, Dane AP, Hallwirth CV, Smyth CM, Wilkie EE, Amaya AK, Zhu E, Khandekar N, Ginn SL, Liao SHY, Cunningham SC, Sasaki N, Cabanes-Creus M, Tam PPL, Russell DW, Lisowski L, Alexander IE: Identification of liver-specific enhancer-promoter activity in the 3' untranslated region of the wild-type AAV2 genome. Nat Genet 2017;49:1268-1273.

-3 Cancer Genome Atlas Research Network. Electronic address wbe, Cancer Genome Atlas Research $\mathrm{N}$ : Comprehensive and Integrative Genomic Characterization of Hepatocellular Carcinoma. Cell 2017;169:1327-1341.

4 El-Khoueiry AB, Sangro B, Yau T, Crocenzi TS, Kudo M, Hsu C, Kim TY, Choo SP, Trojan J, Welling THR, Meyer T, Kang YK, Yeo W, Chopra A, Anderson J, Dela Cruz C, Lang L, Neely J, Tang H, Dastani HB, Melero I: Nivolumab in patients with advanced hepatocellular carcinoma (CheckMate 040): an open-label, noncomparative, phase 1/2 dose escalation and expansion trial. Lancet 2017;389:2492-2502.

5 Chan SL, Wong VW, Qin S, Chan HL: Infection and Cancer: The Case of Hepatitis B. J Clin Oncol 2016;34:8390.

6 Liu Y, Chai Y, Zhang J, Tang J: A Function Variant at miR-501 Alters Susceptibility to Hepatocellular Carcinoma in a Chinese Han Population. Cell Physiol Biochem 2016;38:2500-2508.

7 Wang Y, He L, Du Y, Zhu P, Huang G, Luo J, Yan X, Ye B, Li C, Xia P, Zhang G, Tian Y, Chen R, Fan Z: The long noncoding RNA lncTCF7 promotes self-renewal of human liver cancer stem cells through activation of Wnt signaling. Cell Stem Cell 2015;16:413-425.

$>8$ Lau CC, Sun T, Ching AK, He M, Li JW, Wong AM, Co NN, Chan AW, Li PS, Lung RW, Tong JH, Lai PB, Chan HL, To KF, Chan TF, Wong N: Viral-human chimeric transcript predisposes risk to liver cancer development and progression. Cancer Cell 2014;25:335-349.

-9 Cao C, Sun J, Zhang D, Guo X, Xie L, Li X, Wu D, Liu L: The long intergenic noncoding RNA UFC1, a target of MicroRNA 34a, interacts with the mRNA stabilizing protein HuR to increase levels of beta-catenin in HCC cells. Gastroenterology 2015;148:415-426 e418.

10 Hong X, Kim ES, Guo H: Epigenetic Regulation of Hepatitis B Virus Covalently Closed Circular DNA: Implications for Epigenetic Therapy against Chronic Hepatitis B. Hepatology 2017. Doi:10.1002/hep.29479

11 Yang Y, Chen L, Gu J, Zhang H, Yuan J, Lian Q, Lv G, Wang S, Wu Y, Yang YT, Wang D, Liu Y, Tang J, Luo G, Li Y, Hu L, Sun X, Wang D, Guo M, Xi Q, Xi J, Wang H, Zhang MQ, Lu ZJ: Recurrently deregulated lncRNAs in hepatocellular carcinoma. Nat Commun 2017;8:14421.

12 Li D, Liu X, Zhou J, Hu J, Zhang D, Liu J, Qiao Y, Zhan Q: Long noncoding RNA HULC modulates the phosphorylation of YB-1 through serving as a scaffold of extracellular signal-regulated kinase and YB-1 to enhance hepatocarcinogenesis. Hepatology 2017;65:1612-1627.

-13 Yang F, Zhang L, Huo XS, Yuan JH, Xu D, Yuan SX, Zhu N, Zhou WP, Yang GS, Wang YZ, Shang JL, Gao CF, Zhang FR, Wang F, Sun SH: Long noncoding RNA high expression in hepatocellular carcinoma facilitates tumor growth through enhancer of zeste homolog 2 in humans. Hepatology 2011;54:1679-1689.

14 Yuan JH, Liu XN, Wang TT, Pan W, Tao QF, Zhou WP, Wang F, Sun SH: The MBNL3 splicing factor promotes hepatocellular carcinoma by increasing PXN expression through the alternative splicing of IncRNA-PXNAS1. Nat Cell Biol 2017;19:820-832.

15 Rizvi AH, Camara PG, Kandror EK, Roberts TJ, Schieren I, Maniatis T, Rabadan R: Single-cell topological RNA-seq analysis reveals insights into cellular differentiation and development. Nat Biotechnol 2017;35:551-560.

16 Liu M, Xing LQ Liu YJ: A three-long noncoding RNA signature as a diagnostic biomarker for differentiating between triple-negative and non-triple-negative breast cancers. Medicine (Baltimore) 2017;96:e6222.

17 Abraham JM, Meltzer SJ: Long Noncoding RNAs in the Pathogenesis of Barrett's Esophagus and Esophageal Carcinoma. Gastroenterology 2017;153:27-34. 


\section{Cellular Physiology Cell Physiol Biochem 2017;44:125-132 \begin{tabular}{l|l} 
and Biochemistry POblished online: November 07, 2017 & $\begin{array}{l}\text { O 2017 The Author(s). Published by S. Karger AG, Basel } \\
\text { www.karger.com/cpb }\end{array}$ \\
\cline { 1 - 3 }
\end{tabular} \\ Yuan et al.: Plasma LncRNA in HCC}

18 Ren S, Wang F, Shen J, Sun Y, Xu W, Lu J, Wei M, Xu C, Wu C, Zhang Z, Gao X, Liu Z, Hou J, Huang J, Sun Y: Long non-coding RNA metastasis associated in lung adenocarcinoma transcript 1 derived miniRNA as a novel plasma-based biomarker for diagnosing prostate cancer. Eur J Cancer 2013;49:2949-2959.

19 Tang J, Zhuo H, Zhang X, Jiang R, Ji J, Deng L, Qian X, Zhang F, Sun B: A novel biomarker Linc00974 interacting with KRT19 promotes proliferation and metastasis in hepatocellular carcinoma. Cell Death Dis 2014;5:e1549.

20 Yip TC, Chan HL, Wong VW, Tse YK, Lam KL, Wong GL: Impact of age and gender on risk of hepatocellular carcinoma after hepatitis B surface antigen seroclearance. J Hepatol 2017. Doi:10.1016/j.jhep.2017.06.019

21 Lai Q, Vitale A, Iesari S, Finkenstedt A, Mennini G, Spoletini G, Hoppe-Lotichius M, Vennarecci G, Manzia TM, Nicolini D, Avolio AW, Frigo AC, Cillo U, Graziadei I, Rossi M, Tsochatzis E, Otto G, Ettorre GM, Tisone G, Vivarelli M, Agnes S, Lerut J, European Hepatocellular Cancer Liver Transplant Study G: Intention-totreat survival benefit of liver transplantation in patients with hepatocellular cancer. Hepatology 2017. Doi:10.1002/hep.29342

22 Dutta R, Mahato RI: Recent advances in hepatocellular carcinoma therapy. Pharmacol Ther 2017;173:106117.

23 Devaux Y, Zangrando J, Schroen B, Creemers EE, Pedrazzini T, Chang CP, Dorn GW, 2nd, Thum T, Heymans S, Cardiolinc n: Long noncoding RNAs in cardiac development and ageing. Nat Rev Cardiol 2015;12:415-425.

24 Xu N, Chen F, Wang F, Lu X, Wang X, Lv M, Lu C: Clinical significance of high expression of circulating serum IncRNA RP11-445H22.4 in breast cancer patients: a Chinese population-based study. Tumour Biol 2015;36:7659-7665.

25 Yuan SX, Wang J, Yang F, Tao QF, Zhang J, Wang LL, Yang Y, Liu H, Wang ZG, Xu QG, Fan J, Liu L, Sun SH, Zhou WP: Long noncoding RNA DANCR increases stemness features of hepatocellular carcinoma by derepression of CTNNB1. Hepatology 2016;63:499-511.

26 Zhou M, Zhang XY, Yu X: Overexpression of the long non-coding RNA SPRY4-IT1 promotes tumor cell proliferation and invasion by activating EZH2 in hepatocellular carcinoma. Biomed Pharmacother 2017;85:348-354.

27 Li J, Wang X, Tang J, Jiang R, Zhang W, Ji J, Sun B: HULC and Linc00152 Act as Novel Biomarkers in Predicting Diagnosis of Hepatocellular Carcinoma. Cell Physiol Biochem 2015;37:687-696.

-28 Konishi H, Ichikawa D, Yamamoto Y, Arita T, Shoda K, Hiramoto H, Hamada J, Itoh H, Fujita Y, Komatsu S, Shiozaki A, Ikoma H, Ochiai T, Otsuji E: Plasma level of metastasis-associated lung adenocarcinoma transcript 1 is associated with liver damage and predicts development of hepatocellular carcinoma. Cancer Sci 2016;107:149-154.

29 El-Tawdi AH, Matboli M, Shehata HH, Tash F, El-Khazragy N, Azazy Ael S, Abdel-Rahman O: Evaluation of Circulatory RNA-Based Biomarker Panel in Hepatocellular Carcinoma. Mol Diagn Ther 2016;20:265-277.

- 30 Tang J, Jiang R, Deng L, Zhang X, Wang K, Sun B: Circulation long non-coding RNAs act as biomarkers for predicting tumorigenesis and metastasis in hepatocellular carcinoma. Oncotarget 2015;6:4505-4515.

-31 Thai P, Statt S, Chen CH, Liang E, Campbell C, Wu R: Characterization of a novel long noncoding RNA, SCAL1, induced by cigarette smoke and elevated in lung cancer cell lines. Am J Respir Cell Mol Biol 2013;49:204211. 